\title{
Micromechanical Tuning Elements in a 620-GHz Monolithic Integrated Circuit
}

\author{
Victor M. Lubecke, Associate Member, IEEE, William R. McGrath, Member, IEEE, \\ Philip A. Stimson, Member, IEEE, and David B. Rutledge, Fellow IEEE
}

\begin{abstract}
While monolithic integrated-circuit technology promises a practical means for realizing reliable reproducible planar millimeter and submillimeter-wave circuits, conventional planar circuits do not allow for critical post-fabrication optimization of performance. A 620-GHz quasi-optical monolithic detector circuit is used here to demonstrate the performance of two integrated micromechanical planar tuning elements. This is the first reported demonstration of integrated micromechanical tuning at submillimeter wavelengths. The tuning elements, called sliding planar backshorts (SPB's), are used to adjust the electrical length of planar transmission-line tuning stubs to vary the power delivered between a substrate-lens coupled planar antenna and a thin-film bismuth detector over a range of nearly $15 \mathrm{~dB}$. The circuit performance agrees with theoretical calculations and microwave measurements of a $-0.06-d B$ reflection coefficient made for a scale model of the integrated tuners. The demonstrated tuning range for the SPB tuners indicates that they can be valuable for characterizing components in developmental circuits and for optimizing the inuse performance of various millimeter and submillimeter-wave integrated circuits.
\end{abstract}

Index Terms-Micromachining, submillimeter waves, tuners.

\section{INTRODUCTION}

$\mathbf{S}$ UBMILLIMETER-WAVELENGTH molecular emissions are a valuable resource for providing insight into the chemical makeup and dynamics of stars, the intergalactic medium, and the earth's atmosphere [1], [2]. Over the past two decades, mechanically tunable waveguide circuits have been used to make sensitive spectroscopic measurements in the millimeter and submillimeter-wave bands. In these circuits, a device such as a Schottky-diode or superconductor-insulator-superconductor (SIS) junction is embedded in

Manuscript received January 14, 1998; revised August 10, 1998. This work was supported by the Innovative Science and Technology Office of the Ballistic Missile Defense Organization and by the National Aeronautics and Space Administration, Office of Space Science. The work of V. M. Lubecke was supported in part by NASA under a GSRP Fellowship.

V. M. Lubecke was with the Division of Engineering and Applied Science, California Institute of Technology, Pasadena, CA 91125 USA. He is now with the Photodynamics Research Center, Institute of Physical and Chemical Research (RIKEN), Sendai 980, Japan.

W. R. McGrath is with the Center for Space Microelectronics Technology, Jet Propulsion Laboratory, California Institute of Technology, Pasadena, CA 91109 USA.

P. A. Stimson was with the Center for Space Microelectronics Technology, Jet Propulsion Laboratory, California Institute of Technology, Pasadena, CA, 91109 USA. He is now with the Australian Defence Science and Technology Organization, Salisbury 5108, S.A., Australia.

D. B. Rutledge is with the Division of Engineering and Applied Science, California Institute of Technology, Pasadena, CA, 91125 USA.

Publisher Item Identifier S 0018-9480(98)09060-7. a waveguide mixer block, and waveguide tuning stubs with mechanically adjustable backshorts are used to optimize performance by compensating for parasitic device reactance. Fabrication of such circuits is a difficult and costly procedure, and the steadily decreasing size of higher frequency waveguide features only makes the challenge more severe.

An attractive alternative is the use of all-planar integratedcircuit technology. Such circuits are fabricated through photolithographic techniques alone, making them simpler and more cost-effective than conventionally machined waveguide circuits. A variety of these circuits have been demonstrated using a substrate lens to quasi-optically couple millimeter and submillimeter-wave signals to planar antennas and devices [5]-[7]. This approach can provide better reproducibility and reliability, allow for the creation of focal plane-imaging arrays without an increase in the complexity of the fabrication process [8], and could potentially allow for the inclusion of intermediate frequency (IF) circuitry to form fully integrated receivers. However, device parasitics are still significant [9], [10] and must be compensated for using planar circuit elements, which are typically of fixed form and do not provide a means of post-fabrication optimization. This results in a greater need for accurate device and circuit characterization and optimization through iterative fabrication.

It is desirable to integrate adjustable tuning elements into planar circuits, which would function analogously to the adjustable backshorts found in waveguide circuits, and techniques demonstrated in the fabrication of silicon micromachines suggest a means for realizing them. The anisotropic etching of silicon (bulk micromachining) has been used to form rigid submillimeter-wave antenna [11], [12] and transmission-line structures, [13], [14] and sacrificiallayer techniques (surface micromachining) used to make deforming [15], and movable [16] switches for much lower frequencies. By applying surface micromachining techniques with appropriate materials, in combination with LIGA-like molding and electroforming techniques, movable planar tuning structures can be included in the fabrication of monolithic submillimeter-wave integrated circuits [17].

A mechanically adjustable planar tuning element called a sliding planar backshort (SPB) has been developed, which varies the electrical length of the planar transmission line into which it is integrated. The tuning element is fabricated with a unique implementation of photolithographic micromachining techniques and preserves all of the benefits typically associated with planar integrated circuits. The 


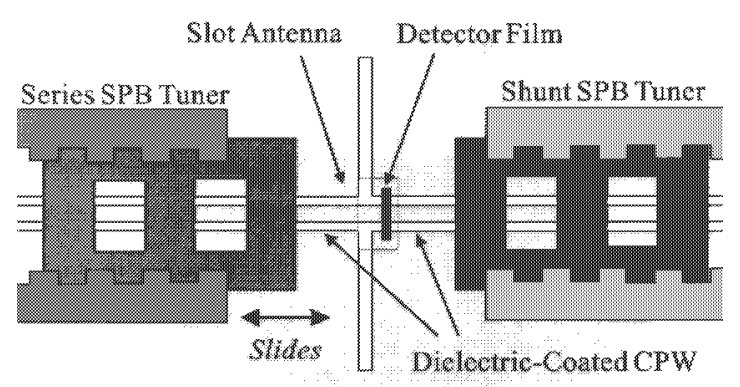

(a)

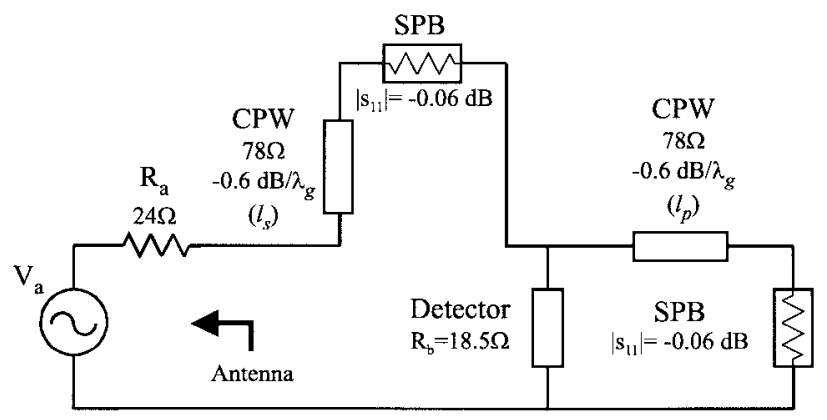

(b)

Fig. 1. (a) Circuit layout and (b) theoretical model [22]-[27] for the tunable $620-\mathrm{GHz}$ integrated circuit. Two SPB tuners are used: one to create a variable series reactance in between the antenna and detector and the other to create a variable susceptance in parallel with the detector.

performance of two SPB tuners is demonstrated here in a fully monolithic integrated circuit, where they are used to adjust the impedance match between a slot antenna and a thin-film detector to predictably vary the circuit response over a range of nearly $15 \mathrm{~dB}$ at $620 \mathrm{GHz}$ [18]. This is the first reported demonstration of integrated micromechanically adjustable tuning at submillimeter wavelengths. Such tuning elements may be used in developmental circuits as an aid to characterizing state-of-the-art devices or to provide real-time performance optimization in a wide range of millimeter and submillimeter-wave integrated circuits.

\section{DESIGN}

A quasi-optical 620-GHz monolithic direct-detection circuit was developed to demonstrate the operation of integrated SPB tuners. This circuit used a dielectric-filled parabola to focus radiation onto a slot antenna and coupled this radiation to a bismuth detector by means of two coplanar waveguide (CPW) transmission lines, each with integrated SPB tuners. One SPB was used to create a variable series reactance between the antenna and detector, potentially serving to compensate for any off-resonance reactance of the slot. The other SPB created a variable susceptance in parallel with the detector, and could act to compensate for the parasitic capacitance found in otherwise desirable submillimeter-wave devices. The integrated circuit design is illustrated in Fig. 1.

An SPB consists of a rectangular metal plate with appropriately sized and spaced holes, which rests on top of a dielectric-coated planar transmission line. The impedance of the sections of line covered by metal is greatly reduced, while

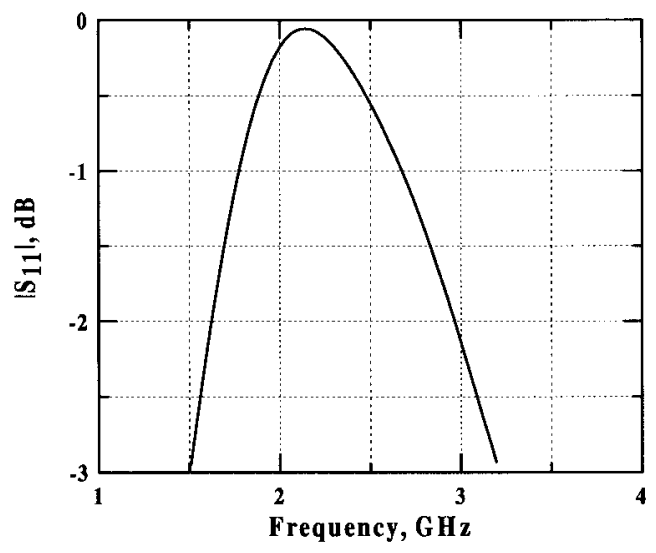

(a)

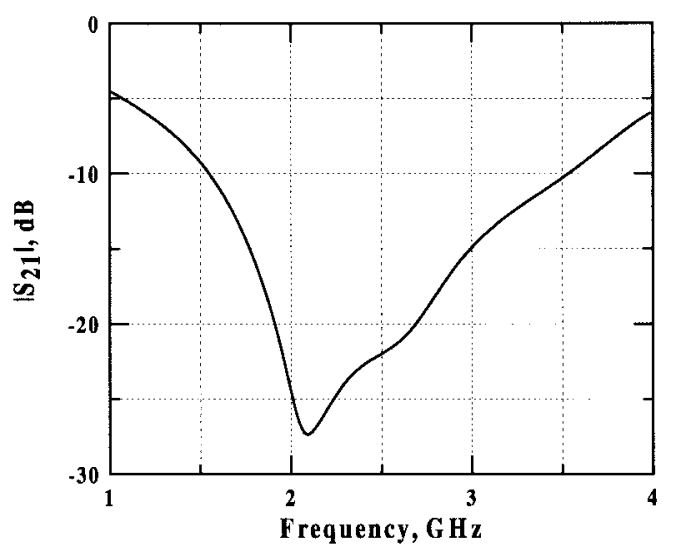

(b)

Fig. 2. Plot of the measured (a) reflection coefficient and (b) transmission coefficient for a scale model of the integrated SPB on a $78-\Omega$ CPW transmission line. Measured $\left|s_{11}\right|$ is better than $-0.5 \mathrm{~dB}$ over a $30 \%$ bandwidth.

the uncovered sections retain their higher impedance. Each of these sections is approximately one quarter-wavelength long, and the cascade of alternating low-impedance and highimpedance sections results in an extremely low-impedance termination at the position along the transmission line at which the plate is positioned. The critical dimensions of the $620-\mathrm{GHz}$ integrated SPB tuners used in this experiment were scaled from a tuning element, which was empirically designed at $2 \mathrm{GHz}$ for use on a 204- $\Omega$ coplanar-strip transmission line [19]. A frequency-scaled version of this tuning element has also been demonstrated at $100 \mathrm{GHz}$ [20]. The 78- $\Omega \mathrm{CPW}$ used here is the physical dual of that transmission line, and the return loss of the SPB in this application was also measured at $2 \mathrm{GHz}$ as $\left|s_{11}\right|=-0.06 \mathrm{~dB}$. Insertion and return loss measurements were made from $20 \mathrm{MHz}$ to $5 \mathrm{GHz}$ using an $\mathrm{HP}$ 8510 network analyzer, and $\left|s_{11}\right|$ remained better than $-0.5 \mathrm{~dB}$ over a bandwidth of approximately 30\%, as shown in Fig. 2. The 620-GHz frequency-scaled SPB consisted of three covered sections, each approximately 80- $\mu \mathrm{m}$ long, and two uncovered sections, approximately 65- and 75- $\mu \mathrm{m}$ long. Additional uncovered and covered sections were added to the trailing end of the SPB to better facilitate its manipulation with a mechanical probe. The width of the exterior of the $620-\mathrm{GHz}$ SPB was 200 $\mu \mathrm{m}$, and the holes were $110-\mu \mathrm{m}$ wide. These dimensions were chosen to avoid lateral resonances at the design frequency. 
The dielectric-filled parabola used in this experiment consisted of a plano-convex fused-quartz lens with the convex surface shaped into a parabola with a focal-length-to-diameter ratio of $1: 4$ [21]. The parabolic side was metalized to function analogously to a conventional parabolic dish antenna, focusing incident paraxial radiation to a small beam-waist. The integrated detector circuit was fabricated on a fused-quartz wafer and positioned on the lens so that the antenna coincided with this beam-waist.

The CPW transmission lines were designed to optimize the effect of the tuning elements, and the antenna was designed to be compatible with the dimensions of the CPW and SPB tuners. The antenna was a full-wave resonant slot, $261-\mu \mathrm{m}$ long, 5- $\mu \mathrm{m}$ wide, and designed to have a feed impedance of $24 \Omega$ at $620 \mathrm{GHz}$ [22]. The $\mathrm{CPW}$ ransmission lines consisted of a $16-\mu \mathrm{m}$-wide center conductor, with $8-\mu \mathrm{m}$-wide gaps on each side, and were designed to have a characteristic impedance of $78 \Omega$ and a guide wavelength $\lambda_{g}$ of $312 \mu \mathrm{m}$ [23], [24]. The line was also designed to minimize loss due to radiation into the substrate [25]. Conductor loss for such a line can be minimized by the use of a highly conductive metal or superconducting film. Total loss for the lines in this experiment was calculated to be $0.6 \mathrm{~dB} / \lambda_{g}$.

For this experiment a small bismuth film was used to create a self-heating thermocouple for detection of the submillimeter radiation. It was patterned to lie across both sides of the CPW line, near the antenna. Current induced in the antenna by a submillimeter signal passes though the film and heats it, and the physical symmetry of the interface between the bismuth and conductors of the CPW results in a thermal-electric voltage, which is proportional to the power absorbed by the film. This circuit was designed to accommodate a four-wire resistance measurement, allowing the bismuth film to be used as a microbolometer as well [26]. The advantage of using the film as a thermocouple is that it requires no bias current and, thus, has reduced noise [27]. Using a bismuth film, which is much thicker than the metal layer with which the detector must overlap, insures good edge coverage and also results in a lowimpedance detector, which closely matches the $24-\Omega$ antenna. The measured dc resistance of the two bismuth detectors (33 and $42 \Omega$ ) appeared in parallel for the RF circuit as $18.5 \Omega$. This impedance match and, consequently, the output of the circuit, could then be altered by varying the positions of the SPB tuners.

\section{EXPERIMENTAL RESULTS}

The seven-layer submillimeter-wave detector circuit was fabricated entirely by monolithic techniques. The antenna, CPW, and detectors were fabricated using standard thin-film techniques. The SPB tuners were fabricated using a unique application of molding and sacrificial-layer techniques. These are similar to techniques which are commonly applied in the micromachining of silicon, but have been adapted to materials and processes suitable for a submillimeter-wave integrated circuit. Simple circuit fabrication facilities lacking dedicated environmental controls proved adequate. The entire circuit was fabricated on a circular 18 -mm-diameter $254-\mu \mathrm{m}$-thick fused-

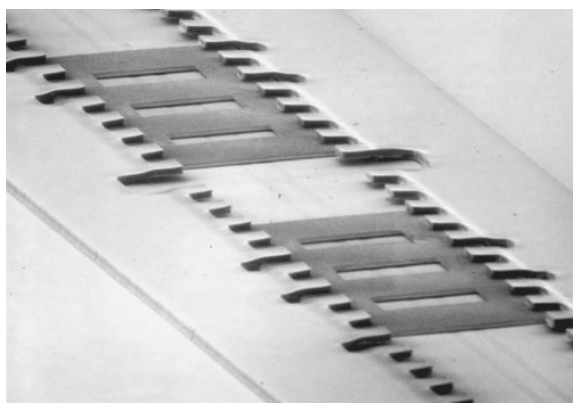

(a)

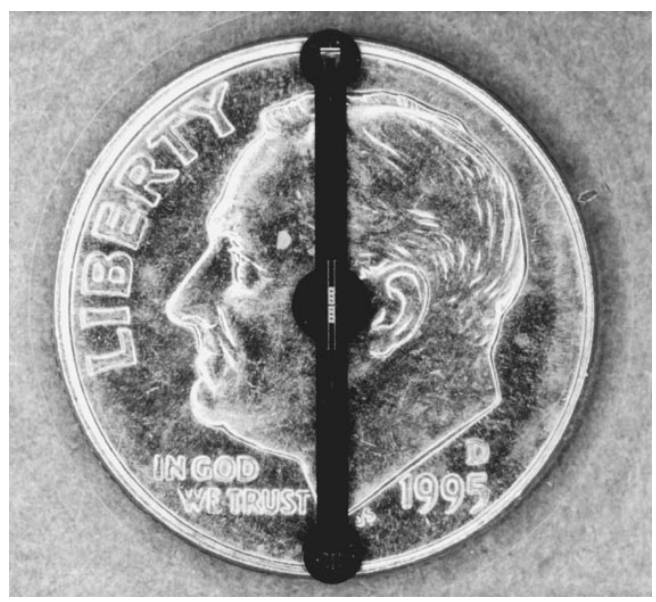

(b)

Fig. 3. (a) An SEM photograph of the integrated SPB tuners and (b) an optical photograph of the entire transparent circuit wafer compared with a dime. Each SPB is $200-\mu \mathrm{m}$ wide and $5-\mu \mathrm{m}$ thick and slides along a polyimide guide structure to provide tuning. The fused-quartz wafer is placed on a substrate lens of the same material to quasi-optically couple to a $620-\mathrm{GHz}$ signal.

quartz wafer. Conventionally patterned thin-films were used for the dielectric-coated transmission lines and antenna, and the SPB tuners added over a sacrificial seed layer through two metal electrodepositions and a spin coating of polyimide [28], [29]. After removing the sacrificial metal to allow the patterned metal elements to slide freely within the polyimide guides, a patterned thin-film of bismuth was added as the detector. An SEM photograph of the center of the circuit is shown in Fig. 3(a), and a photograph of the entire circuit is shown in Fig. 3(b).

The circuit was mounted in a brass fixture over a recess containing the fused-quartz parabola with gold-film backing. Aluminum bond wires were used to connect the center and outer conductors on each end of the CPW to individual printed circuit boards on the mount, each terminated with a connector. This was done in order to allow various connection methods to be tried for measurement of the detected signal. The fixture was attached (with the circuit facing upwards) to an adjustable gimbal mount on top of two orthogonal lineartranslation stages mounted on an optical-measurement table. A gold mirror mounted on translational and rotational stages was used to direct a horizontally incident signal onto the circuit. A microscope with a magnification of $1000 \times$ was positioned at an angle above the circuit to aid in the manipulation of the SPB tuners. The setup is illustrated in Fig. 4(a). 


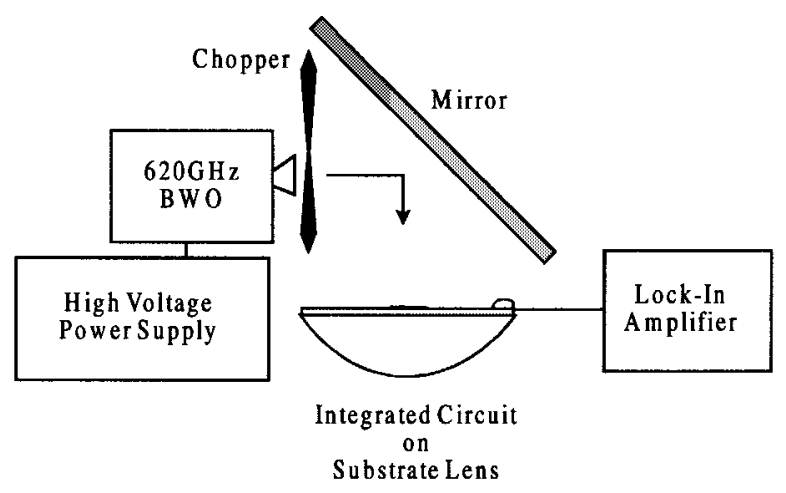

(a)

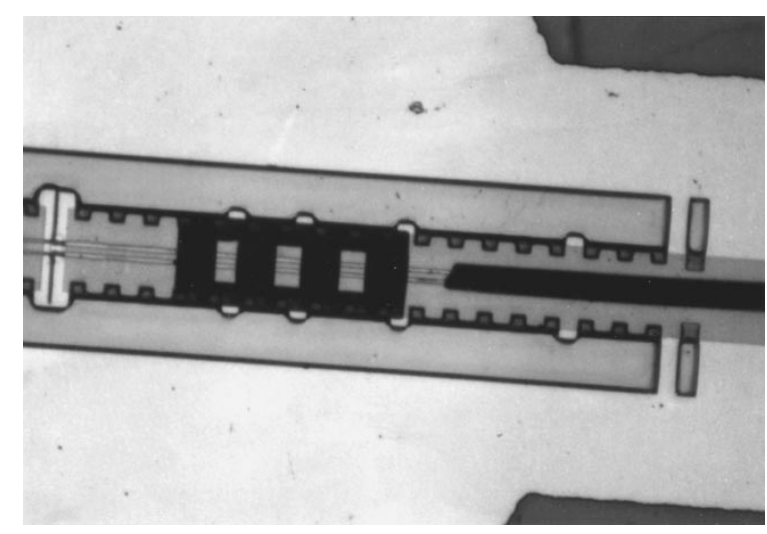

(b)

Fig. 4. Illustration of the (a) 620-GHz measurement system and (b) microscope photograph of SPB tuner manipulation. A mirror was used to direct the chopped signal onto the substrate lens of the circuit with a lock-in amplifier used to monitor the circuit response while the SPB tuners were manipulated with an ox-hair probe.

A backward-wave oscillator (BWO) was used as a $620-\mathrm{GHz}$ source. This source provided a few milliwatts of multimode power with much less than $1 \%$ in the fundamental mode, which couples to the circuit. It was positioned behind a $25-\mathrm{Hz}$ chopper and as close as possible to the gold mirror. A PAR$125 \mathrm{~A}$ lock-in amplifier was used to measure the output voltage of the detector. The system was first aligned to maximize the detected signal (approximately $2 \mu \mathrm{V}$ ) with the tuning elements in somewhat arbitrary positions. The voltage was measured across the two detectors in series.

A probe was fashioned with a $50-\mu \mathrm{m}$-diameter ox hair at its tip and used to manually position the appropriate SPB for each measurement, as shown in Fig. 4(b). Micrometerdriven positioners were not used here due to mechanical constraints, but could provide improved usability in most circuits. Manual adjustments proved adequate here for the desired $\lambda_{g} / 16$ position increments.

Measurements were made of the power delivered to the detector as the SPB tuners were adjusted. The measured data obtained by sweeping the position of the series tuning element incrementally over a distance of one guide-wavelength for a fixed position of the parallel tuning element is shown in Fig. 5. Data sets obtained by sweeping the parallel tuning element incrementally over a range of three guide-wavelengths for two

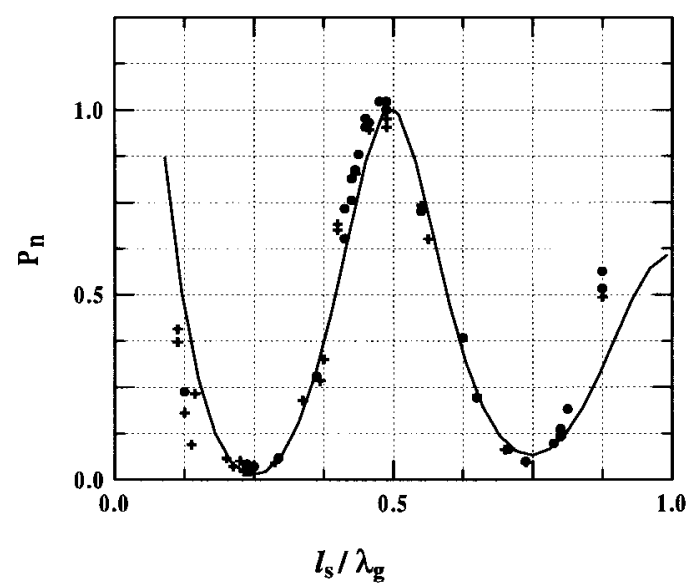

Fig. 5. Measured $(\bullet)(+)$ and theoretical $(-)$ response for series tuning of the $620-\mathrm{GHz}$ detector circuit with the parallel tuner in a fixed position $\left(l_{p}=0.321 \lambda_{g}\right)$. The power absorbed by the detector $P_{n}$ is shown as a function of the series tuner position, $l_{s}$ normalized to that measured near the peak with $l_{s}=0.488 \lambda_{g}$.

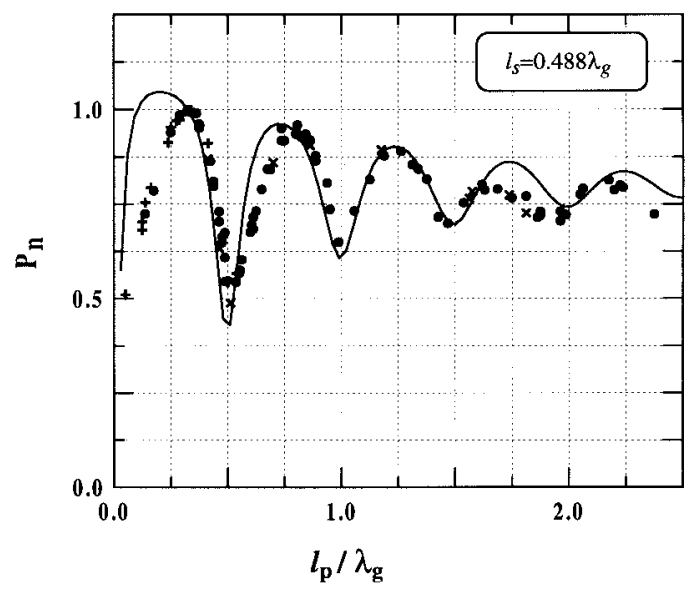

(a)

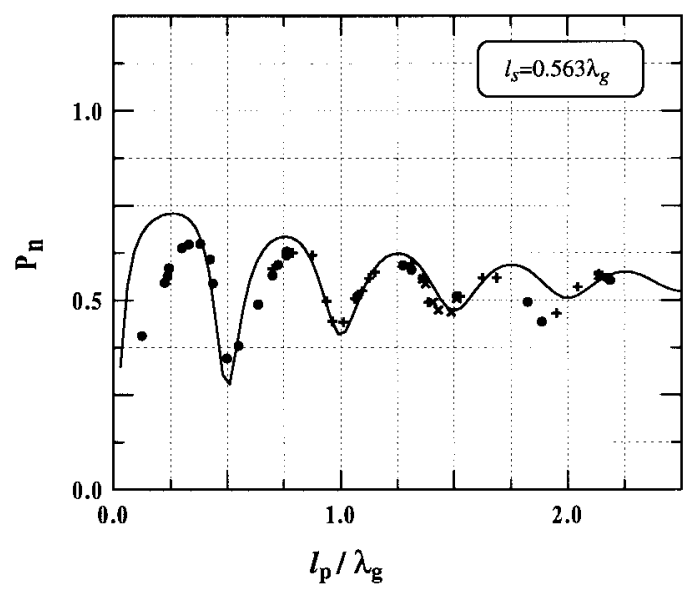

(b)

Fig. 6. Measured $(\bullet)(+)$ and theoretical $(-)$ response for shunt tuning of the $620-\mathrm{GHz}$ detector circuit, with the series tuner in two different fixed positions (a) and (b). The power absorbed by the detector $P_{n}$ is shown as a function of the parallel tuner position $l_{p}$ normalized to that measured near the peak with $l_{p}=0.321 \lambda_{g}$ and $l_{s}=0.488 \lambda_{g}$. 


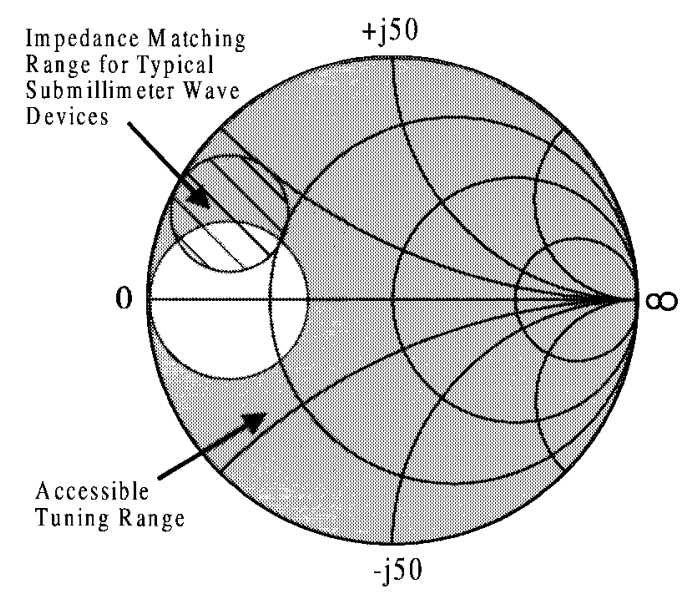

Fig. 7. Smith chart showing the range of impedances to which the integrated SPB tuning circuit can transform a $24-\Omega$ antenna. The indicated range, based on a lossless system, includes impedances suitable for matching to those of SIS and Schottky devices.

different positions of the series tuning element are shown in Fig. 6. These results were recorded over several experimental runs spaced some hours apart, and different symbols have been plotted to represent groups of data recorded in each run. Data for each sweep were normalized to a reference measurement taken near the peak response with the SPB which created the parallel susceptance positioned near $\lambda_{g} / 4$ and the SPB which created the series reactance positioned near $\lambda_{g} / 2$. In each measurement sweep, the SPB tuners functioned to vary the power through multiple peaks and nulls in a repeatable manner.

A theoretical model for the planar circuit, shown in Fig. 2(b), was created to predict and validate the performance of the SPB tuners [30]. The power delivered to the detector $P_{n}$ was calculated as a function of SPB positions $l_{s}$ (for the $\mathrm{SPB}$ in series between the antenna and detectors) and $l_{p}$ (for the SPB in parallel with the detectors) normalized to that calculated for the circuit with $l_{s}=0.488 \lambda_{g}$ and $l_{p}=0.321 \lambda_{g}$ corresponding to the positions for the measurement used to normalize the measured data. The theoretical model included impedance and losses calculated for the transmission lines [23]-[25], the dc conductivity measured for the metal film, impedance calculated for the antenna [22], and reflection coefficient measured for the SPB tuners at $2 \mathrm{GHz}$. The theoretical response has been included with the measured data in Figs. 5 and 6.

The results are consistent with those calculated from the model. There may have been some additional coupling phenomena between the SPB and the antenna, particularly for distances of less than $\lambda_{g} / 2$. The theoretical range of detector impedances for which this circuit can provide an impedance match to a $24-\Omega$ antenna is shown in Fig. 7 , which coincides with that of many common submillimeter-wave devices.

\section{CONCLUSIONS}

This paper demonstrates the function of micromechanical tuning elements in a fully monolithic submillimeter-wave integrated circuit. Two micromechanical SPB tuners were integrated with CPW transmission lines in a quasi-optical detector circuit and their performance measured at $620 \mathrm{GHz}$. The circuit performance was consistent with a theoretical model, indicating that each SPB had a reflection coefficient of approximately $\left|s_{11}\right|=-0.06 \mathrm{~dB}$. The tuning elements were used to vary the power delivered to the detector over a range of almost $15 \mathrm{~dB}$ by adding a variable reactance in series with the antenna and a variable susceptance in parallel with the detector. The tuner design can be easily scaled for use at higher (or lower) frequencies, and potentially include microelectromechanical actuation. Fabrication of the SPB tuners involves processes and materials common to and compatible with those typically used in the production of millimeterwave and submillimeter-wave integrated circuits. Such tuning elements are useful as an aid for the characterization of state-of-the-art planar submillimeter-wave devices and for the optimization of performance in a wide range of millimeter and submillimeter-wave monolithic integrated circuits.

\section{ACKNOWLEDGMENT}

The authors would like to thank Y.-C. Tai for valuable discussions on micromachining, P. H. Siegel for providing advice and facilities, including the dielectric-filled parabola, and O. Borić-Lubecke for assistance with the manuscript.

\section{REFERENCES}

[1] T. G. Phillips and J. Keene, "Submillimeter astronomy (heterodyne spectroscopy)," Proc. IEEE, vol. 80, pp. 1662-1678, Nov. 1992.

[2] J. W. Waters, "Submillimeter-wavelength heterodyne spectroscopy and remote sensing of the upper atmosphere," Proc. IEEE, vol. 80, pp. 1679-16701, Nov. 1992.

[3] G. de Lange et al., "Quantum limited responsivity of a $\mathrm{Nb} / \mathrm{Al} 2 \mathrm{O} 3 / \mathrm{Nb}$ SIS waveguide mixer at 460 and first results at 750 and $840 \mathrm{GHz}$," in 4th Int. Symp. Space Terahertz Technol., Los Angeles, CA, Mar. 1993, pp. 41-49.

[4] B. N. Ellison, M. L. Oldfield, D. N. Matheson, B. J. Maddison, C. M. Mann, and A. F. Smith, "Manufacture and test of a $2.5 \mathrm{THz}$ corrugated feedhorn," in 5th Int. Symp. Space Terahertz Technol., Ann Arbor, MI, May 1994, pp. 851-860.

[5] J. Zmuidzinas, H. G. Leduc, J. A. Stern, and S. R. Cypher, "Two junction tuning circuits for submillimeter SIS mixers," IEEE Trans. Microwave Theory Tech., vol. 42, pp. 698-706, Apr. 1994.

[6] S. S. Gearhart and G. M. Rebeiz, "A monolithic $250 \mathrm{GHz}$ Schottkydiode receiver," IEEE Trans. Microwave Theory Tech., vol. 42, pp. 2505-2511, Dec. 1994.

[7] M. Kim, V. M. Lubecke, S. C. Martin, R. P. Smith, and P. H. Siegel, "A dielectric-filled-parabola-feed frequency multiplier," IEEE Microwave Guided Wave Lett., vol. 7, pp. 60-62, Mar. 1997.

[8] P. A. Stimson, R. J. Dengler, H. G. LeDuc, and S. R. Cypher et al., "A planar quasi-optical SIS receiver," IEEE Trans. Microwave Theory Tech., vol. 14, pp. 609-615, Apr. 1993.

[9] I. Mehdi and P. H. Siegel, "Effect of parasitic capacitance on the performance of planar subharmonically pumped Schottky diode mixers," in 5th Int. Symp. Space Terahertz Technol., Ann Arbor, MI, May 1994, pp. 379-393.

[10] R. Blundell and C.-Y. E. Tong, "Submillimeter receivers for radio astronomy," Proc. IEEE, vol. 80, pp. 1702-1720, Nov. 1992.

[11] D. B. Rutledge, S. E. Schwartz, T. L. Hwang, D. J. Angelakos, K. K. Mei, and S. Yokota, "Antennas and waveguides for far-infrared integrated circuits," IEEE J. Quantum Electron., vol. 16, pp. 508-516, May 1980.

[12] W. Y. Ali-Ahmad, G. M. Rebeiz, H. Davé, and G. Chin, "802 GHz integrated horn antennas imaging array," Int. J. Infrared Millim. Waves, vol. 12, no. 5, pp. 481-486, May 1991.

[13] W. R. McGrath, C. K. Walker, M. Yap, and Y.-C. Tai, "Silicon micromachined waveguides for millimeter-wave and subillimeter-wave frequencies," IEEE Microwave Guided Wave Lett., vol. 3, pp. 61-63, Mar. 1993. 
[14] T. M. Weller, L. P. B. Katehi, and G. M. Rebeiz, "A 250-GHz microshield bandpass filter," IEEE Microwave Guided Wave Lett., vol. 5, pp. 153-155, May 1995.

[15] C. Goldsmith, T.-H. Lin, B. Powers, W.-R. Wu, and others, "Micromechanical membrane switches for microwave applications," in IEEE MTT-S Int. Microwave Symp., vol. 1, Orlando, FL, June 1995, pp. 91-94.

[16] L. E. Larson, R. H. Hacket, M. A. Melendes, and R. F. Lohr, "Micromachined microwave actuator (MIMAC) technology-A new tuning approach for microwave integrated circuits," in IEEE Microwave Millimeter-Wave Monolithic Circuits Symp., Boston, MA, June 1991, pp. 27-30.

[17] V. M. Lubecke, W. R. McGrath, and D. B. Rutledge "Millimeter wave performance of a sliding planar backshort," in SPIE Int. Conf. Millimeter Submillimeter Waves Applicat., San Diego, CA, Jan. 1994, pp. 543-544.

[18] V. M. Lubecke, W. R. McGrath, P. A. Stimson, and D. B. Rutledge, "Performance of micromechanical tuning elements in a $620 \mathrm{GHz}$ monolithic integrated circuit," in 7th Int. Symp. Space Terahertz Technol., Charlottesville, VA, Mar. 1996, pp. 220-223.

[19] V. M. Lubecke, W. R. McGrath, and D. B. Rutledge, "Sliding backshorts for planar circuits," Int. J. Infrared Millim. Waves, vol. 12, no. 12, pp. 1387-1397, Dec. 1991

[20] _ "A 100-GHz coplanar strip circuit tuned with a sliding planar backshort," IEEE Microwave Guided Wave Lett., vol. 3, pp. 441-443, Dec. 1993.

[21] P. H. Siegel and R. J. Dengler, "The dielectric-filled parabola: A new millimeter and submillimeter-wavelength receiver/transmitter front end," IEEE Trans. Antennas Propagat., vol. 39, pp. 40-47, Jan. 1991.

[22] G. V. Eleftheriades and G. M. Rebeiz, "Self and mutual admittance of slot antennas on a dielectric half-space," Int. J. Infrared Millim. Waves, vol. 14 , no. 10 , Oct. 1993

[23] K. C. Gupta, R. Garg, and I. J. Bahl, Microstrip Lines and Slotlines. Norwood, MA: Artech House, 1979.

[24] G. Hasnain, A. Dienes, and J. R. Whinnery, "Dispersion of picosecond pulses in coplanar transmission lines," IEEE Trans. Microwave Theory Tech., vol. 34, pp. 738-741, June 1986.

[25] M. Y. Frankel, S. Gupta, J. A. Valdmanis, and G. M. Mourou, "Terahertz attenuation and dispersion characteristics of coplanar transmission lines," IEEE Trans. Microwave Theory Tech., vol. 39, pp. 910-916, June 1991.

[26] T.-L. Hwang, S. E. Schwartz, and D. B. Rutledge, "Microbolometers for infrared detection," Appl. Phys. Lett., vol. 34, no. 11, pp. 730-776, June 1979.

[27] D. P. Neikirk and D. B. Rutledge, "Self-heated thermocouples for farinfrared detection," Appl. Phys. Lett., vol. 41, no. 5, pp. 400-402, Sept. 1992.

[28] V. M. Lubecke, W. R. McGrath, Y.-C. Tai, and D. B. Rutledge, "Microfabrication of linear translator tuning elements in submillimeter-wave integrated circuits," IEEE J. Microelectromech. Syst., to be published.

[29] V. M. Lubecke, W. R. McGrath, and D. B. Rutledge, "Micromechanical sliding planar backshorts," in Int. Semiconductor Device Res. Symp., Charlottesville, VA, Dec. 1995, pp. 477-480.

[30] V. M. Lubecke, "Micromechanical tuning elements for submillimeter wave integrated circuits," Ph.D. dissertation, Dept. Elect. Eng., California Inst. Technol., Pasadena, CA, July 1995.

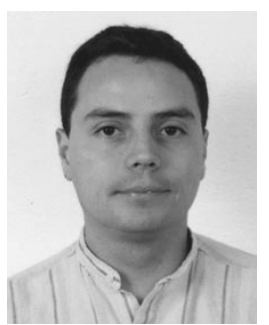

Victor M. Lubecke (S'86-M'86-A'95) received the B.S. degree in electrical and electronic engineering from the California State Polytechnical University, Pomona, in 1986, and the M.S. and $\mathrm{Ph} . \mathrm{D}$. degrees in electrical engineering from the California Institute of Technology, Pasadena, CA, in 1990 and 1995, respectively.

From 1987 to 1996, he worked at the NASA Jet Propulsion Laboratory, Pasadena, CA, where he was involved with millimeter and submillimeter-wave technology in space communications and remote sensing applications. Since 1996, he has been a Visiting Researcher at the Photodynamics Research Center, Institute for Physical and Chemical Research (RIKEN), Sendai, Japan. His current research interests include high-frequency integrated circuits, quasi-optics, and related MEMS techniques.

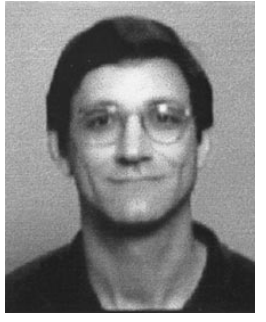

William R. McGrath (M'88) received the B.S degree in physics from the Massachusetts Institute of Technology, Cambridge, in 1978, and the M.A., and $\mathrm{Ph} . \mathrm{D}$. degrees in physics from the University of California at Berkeley in 1981 and 1985, respectively.

$\mathrm{He}$ and his co-workers were the first to measure the quantum effects of large-gain and quantumlimited noise in SIS mixers at $36 \mathrm{GHz}$. Upon graduating from the University of California at Berkeley, he took a position as a Visiting Researcher at the Chalmers University of Technology, Goteborg, Sweden, where he worked on submillimeter-wave superconductive detectors, and where he and co-workers built the first millimeter-wave Josephson effect mixer using the newly discovered high- $T c$ superconductors. In 1987, he joined the staff of the Jet Propulsion Laboratory, Pasadena, CA, where he became a Group Leader in 1990, and a Technical Group Supervisor in 1992. He currently heads a research group, which develops high-performance submillimeter-wave sensors for remote-sensing applications. He has authored or co-authored over 120 publications, and holds two patents.

Dr. McGrath is the recipient of several awards related to his research activities.

Philip A. Stimson (M'92) received the B.Sc. degree with honours from the University of New England, Armidale, N.S.W., Australia, in 1982, and the $\mathrm{Ph} . \mathrm{D}$. degree from the University of Sydney, Sydney, N.S.W., Australia, in 1987.

During 1987, he worked with the CSIRO Division of Applied Physics, Sydney, N.S.W., Australia. In 1988, he held a CSIRO Post-Doctoral Fellowship at the California Institute of Technology, Pasadena, was a Caltech Research Fellow in 1989, and worked at the Jet Propulsion Laboratory, Pasadena, CA, from 1990 to 1996 . He is currently with the Australian Defence Science Technology Organization, Salisbury, S.A., Australia. His interests include millimeter and submillimeter-wave optical systems, antennas and receivers, and satellite communications.

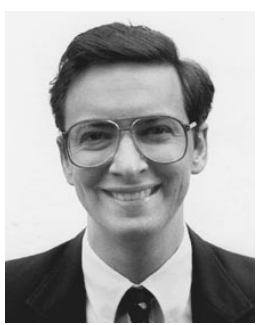

David B. Rutledge (S'77-M'77-SM'89-F'93) received the B.A. degree in mathematics from Williams College, Williamstown, MA, in 1973, the M.A. degree in electrical sciences from Cambridge University, Cambridge, U.K., in 1975, and the $\mathrm{Ph} . \mathrm{D}$. degree from the University of California at Berkeley, in 1980

He has been teaching at the California Institute of Technology, Pasadena, and working on microwave circuits and antennas since 1980. His research group developed key ideas in integrated-circuit antennas, including lens-coupled antennas, which appear widely in radio-astronomy receivers. His group demonstrated anisotropic etching for fabricating horns and membrane technology for suspending metal antennas, first described leakage from planar transmission lines, and first demonstrated many active quasi-optical components, including phase shifters, oscillators, mixers and amplifiers. Recently, his group has developed Class-E high-frequency power amplifiers for industrial and amateur use. He has authored or co-authored over 200 publications. He co-authored the computer-aided design package "Puff," with 15000 copies distributed worldwide. In the summer of 1985 , he was a Visiting Scientist at CSIRO, Sydney, N.S.W., Australia. In the spring and summer of 1988, he was with the Research Institute for Electrical Communication, Tohoku University, Sendai, Japan. In the fall of 1995, he was with the National Defense Academy, Yokosuka, Japan.

Dr. Rutledge is a member of the IEEE AP-S AdCom. From 1991 to 1993, he was a distinguished lecturer for the IEEE Antennas and Propagation Society. He has served as chairman for 19 doctoral candidates. He was honored with the Teaching Award of the Associated Students of Caltech, and five of his students have been awarded Presidential Investigator and Career Awards. He was a recipient of the IEEE Microwave Theory and Techniques Society Microwave Prize in 1993. 